\title{
Optimizing the Nion STEM for In-Situ Experiments
}

\author{
Michael T. Hotz ${ }^{1}$, George Corbin ${ }^{1}$, Niklas Dellby ${ }^{1}$, Tracy C. Lovejoy ${ }^{1}$, Gwyn S. Skone ${ }^{1}$, Jean-Denis \\ Blazit $^{2}$, Mathieu Kociak ${ }^{2}$, Odile Stephan ${ }^{2}$, Marcel Tencé ${ }^{2}$, H.W. Zandbergen ${ }^{3}$ and Ondrej L. Krivanek ${ }^{1,4}$ \\ 1. Nion R\&D, 11511 NE 118th St., Kirkland, WA 98034, USA \\ 2. Laboratoire de Physique des Solides, CNRS, Université Paris Sud, 91405 Orsay, France \\ 3. Department of NanoScience, Delft University of Technology, 2628 CJ Delft, The Netherlands \\ 4. Department of Physics, Arizona State University, Tempe, AZ 85287, USA
}

Designers of electron microscopes are often pulled in two directions:

a) maximizing the performance, such as the microscope's spatial resolution, energy resolution, beam current, and stability,

b) maximizing the flexibility, i.e. the microscope's ability to perform a variety of experiments beyond imaging and diffraction, such as in-situ/operando microscopies, and various spectroscopies.

The two directions are often in conflict, e.g. reaching optimum imaging performance demands small objective lens (OL) gaps, stable temperatures, and excellent vacuum, whereas maximum flexibility demands large OL gaps, variable temperatures, and sometimes also elevated pressures. Here we describe our approach to combining high performance with operational flexibility and show experimental results from a side-entry Nion high-energy resolution monochromated EELS (electron energy loss spectroscopy)

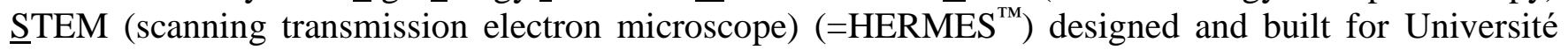
Paris-Sud in Orsay.

The microscope includes Nion's ground potential monochromator and a Nion EEL spectrometer. It has been able to achieve $5 \mathrm{meV}$ full width half maximum (FWHM) of the EELS zero loss peak at $\mathrm{E}_{\mathrm{o}}=30 \mathrm{keV}$ and $6.0 \mathrm{meV}$ at $\mathrm{E}_{\mathrm{o}}=60 \mathrm{keV}$ [1]. The microscope will specialize in high energy resolution optical spectroscopies such as cathodoluminescence (CL) [2], complemented by ultra-high resolution EELS. In order to accommodate an efficient ( $>3$ sr collection angle) CL mirror, the OL gap has been increased to 6 $\mathrm{mm}$. This poses the risk of worsened spatial resolution due to a larger coefficient of chromatic aberration $\left(\mathrm{C}_{\mathrm{c}}\right)$. However, chromatic blurring of STEM images can be overcome by moderate monochromation (from $\sim 0.35 \mathrm{eV}$ energy spread to $\sim 0.1 \mathrm{eV}$ ) [3], and the impact of the increased OL gap on the attainable spatial resolution is therefore expected to be minimal.

The side entry stage, described previously [4], has been designed for optimum mechanical stability and also for ultra high vacuum (UHV). To achieve UHV, we only use metal seals in the microscope column or double O-rings with ion pumped guard vacuum in the interspace and are careful about not exposing Orings that normally reside in the microscope's high vacuum volume to air. As a result, we are able to reach a sample chamber vacuum better than $1 \times 10^{-8}$ Torr within minutes of inserting a new sample holder. The vacuum approached after overnight pumping (without baking) is in the $10^{-10}$ Torr range.

Sample holders developed for the microscope so far include single tilt, double tilt, and a variable temperature single tilt cooling holder that uses a MEMS chip and can operate at temperatures from liquid nitrogen $\left(\mathrm{LN}_{2}\right)$ to about $1000^{\circ} \mathrm{C}$. Other holders including a double-tilt cooling holder and a cryotransfer holder are under development, and future plans include further holders such as liquid and gas cell ones. 
The performance of the cooling holder has been exceptional: it routinely reaches less than $1.2 \AA$ resolution at its lowest temperature (Fig. 1), which it can hold for several hours. Because of the dry UHV in the Nion column, no $\mathrm{LN}_{2}$-cooled cryoshield is needed to keep the sample from frosting up with ice.

As an interesting demonstration experiment, we performed spectroscopy on ice. The vacuum in the sample chamber was too high to condense ice on the cooled sample, and we therefore introduced a moisture reservoir (wet O-ring) into the sample airlock and cooled the sample in the airlock in a poor vacuum of low-pressure $\mathrm{H} 2 \mathrm{O}$ vapor. Ice layers grown this way remained stable inside the microscope, neither growing nor shrinking except when irradiated. We were able to collect vibrational spectra of ice in a damage-avoiding way, by using a stationary beam that made a hole in the ice and acquiring spectra from the surrounding undamaged ice in an aloof scattering geometry (Fig. 2).

In summary, combining a careful STEM column design with high energy resolution monochromation, ultra-high energy resolution EELS, a flexible and stable side-entry stage, a variety of sample holders, and an efficient mirror plus a CL spectrometer (and a laser illumination system) provides a very flexible experimental platform with no substantial performance penalties. We look forward to this system advancing electron microscopy in many different directions [5].

[1] T.C. Lovejoy et al., these proceedings.

[2] M. Kociak and L.F. Zagonel, Ultramicroscopy 176 (2017) 112-131.

[3] O.L. Krivanek et al., these proceedings.

[4] M.T. Hotz et al., Microsc. Microanal. 23 (Suppl 1), 2017, p. 54-55.

[5] This work has received support from the French National Agency for Research under the program of future investment TEMPOS- CHROMATEM, reference No. ANR-10- EQPX- 50.

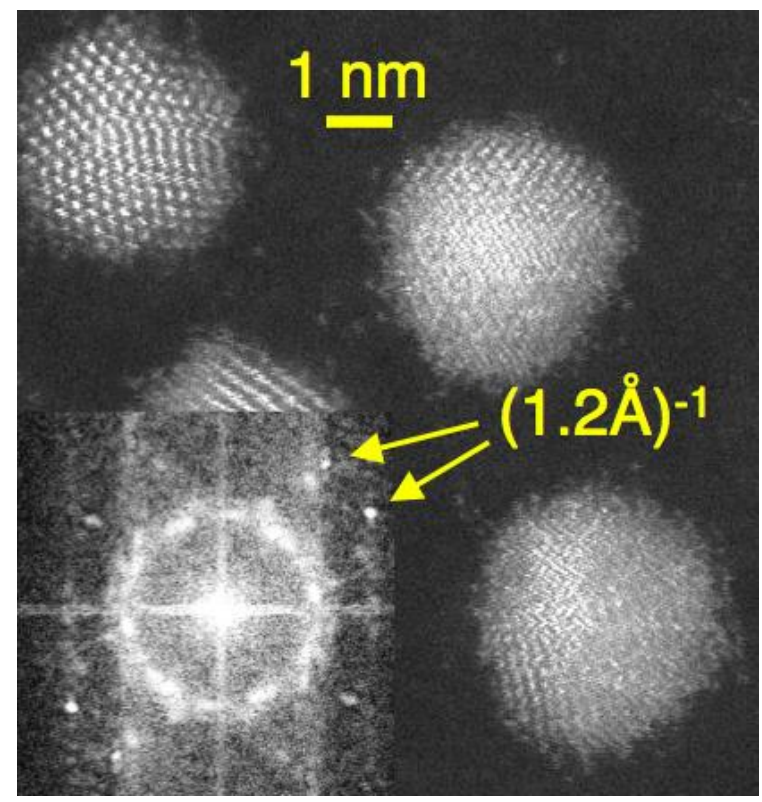

Figure 1. Gold particles imaged at liquid N2 temperature at $200 \mathrm{keV}$. The FFT insert shows strong spots at $(1.2 \AA)^{-1}$, and single atoms are resolved between the particles.

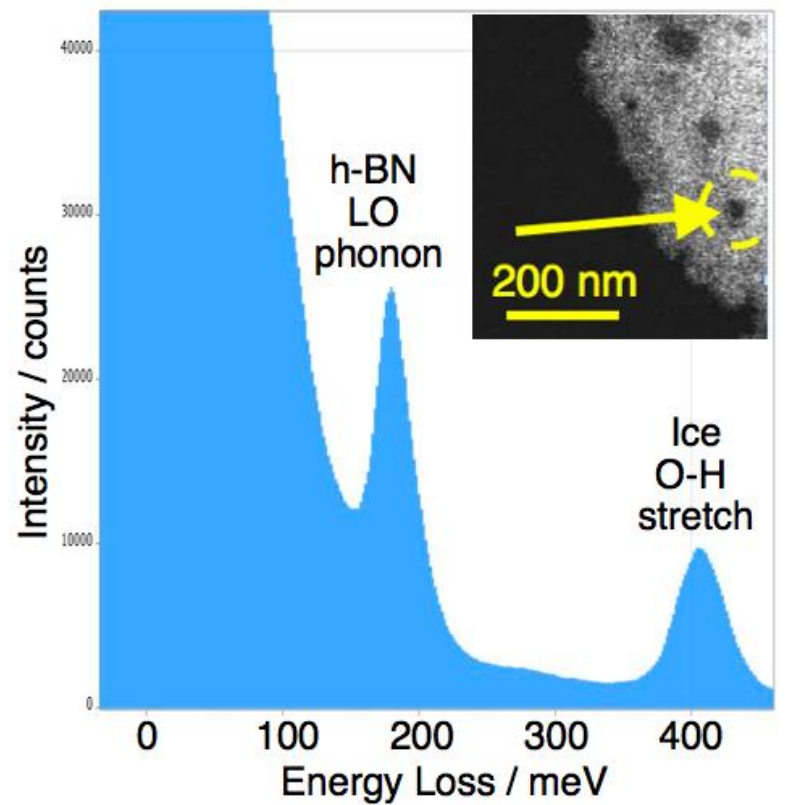

Figure 2. Vibrational spectrum (acquired at 100 $\mathrm{keV}$ ) of ice adsorbed onto an h-BN flake. Insert shows the h-BN flake, coated with about $30 \mathrm{~nm}$ of ice, with a hole made by the beam (arrowed). 